\title{
Inhibitory Potential of Herbal Medicines on Human Cytochrome P450- Mediated Oxidation: Properties of Umbelliferous or Citrus Crude Drugs and Their Relative Prescriptions
}

\author{
Lian-Qing Guo ${ }^{1,4}$, Masahiko Taniguchi², Qiao-Yun Chen $^{3}$, Kimiye Baba ${ }^{2}$ and Yasushi Yamazoe ${ }^{1, *}$ \\ ${ }^{I}$ Division of Drug Metabolism and Molecular Toxicology, Graduate School of Pharmaceutical Sciences, Tohoku University, \\ Sendai 980-8578, Japan \\ ${ }^{2}$ Division of Pharmacognosy, Osaka University of Pharmaceutical Sciences, Takatsuki 569-1094, Japan \\ Departments of ${ }^{3}$ Pharmacy and ${ }^{4}$ Clinical Pharmacology, Jinling Hospital, Nanjing 210002, China
}

Received November 27, 2000 Accepted January 16, 2001

\begin{abstract}
To investigate the possible drug interaction with herbal medicine, hot water decoctions or $40 \%$ ethanol infusions of several Umbelliferous or Citrus crude drugs and their prescriptions were examined in vitro for their abilities to inhibit human cytochrome P450 3A (CYP3A). Addition of each decoction or infusion from Baizhi (Angelica dahurica and varieties), Qianghuo (Notopterygium incisum or N. forbesii), Duhuo (Angelica biserrata), Fangfeng (Saposhnikovia divaricata), Danggui (Angelica sinensis), Zhishi or Zhiqiao (Citrus aurantium) resulted in various degrees of human CYP3A inhibition as determined by microsomal testosterone $6 \beta$-hydroxylation. The inhibitory potency was consistent with the abundance of the hydrophobic components for each sample. Experiments on the infusion of a Japanese Baizhi (BZ1) showed the major role of furanocoumarins on human CYP3A inhibition. Some of the crude drugs and a related prescription showed increased inhibition after the preincubation, suggesting the involvement of a mechanismbased inhibition. Some formulated prescriptions, however, showed intense inhibition with their hydrophobic fractions rather than with their hydrophobic fractions, suggesting that components other than furanocoumarins in herbal prescriptions may also cause CYP3A inhibition. These results indicate the necessity of intensive investigations on the possible drug interaction with traditional medicines.
\end{abstract}

Keywords: Furanocoumarin, Crude drug, Traditional prescription, Cytochrome P450 3A, Inhibition

Herbal medicines are major parts of traditional medicines. These attract increased attention because of their natural origin and accumulated evidence during years of application. Natural origin does not, however, mean harm free. Herbal medicines may sometimes evoke indirect or direct health risks as do modern or industrized medicines (1). Traditional medicines are often applied for long term therapies, which are inevitable from coadministration of other drugs. Simultaneous use of multiple drugs enhanced the chances of possible pharmacodynamic and/or pharmacokinetic interaction. Changes in drug concentrations in vivo, as well as direct pharmacological interactions at the levels of a molecular receptor, influence drug efficacies and/or induce side effects.

Although influences of traditional medicines on drug metabolism are necessary prior to understand their role in

*Corresponding author. FAX: +81-22-217-6826

E-mail: yamazoe@mail.cc.tohoku.ac.jp drug interaction, only limited numbers of studies have been conducted $(2-6)$. As a typical example for the influence of a natural product on human drug metabolism, the beverage grapefruit juice is known to alter bioavailabilities of many clinically important drugs through its inhibition of cytochrome P450 3A (CYP3A4) in humans (7). Recent studies on the role of furanocoumarin as the causative components in grapefruit juice for CYP3A4 inhibition $(8-11)$ prompts us to examine the other derivatives as possible causative agents for drug interaction.

Naturally, various derivatives of furanocoumarins are detectable in plants belonging to Umbelliferae, Rutaceae, Moraceae and Leguminosae (12). Some furanocoumarins from plants have been developed and are used as conventional drugs for treatment of dermatological diseases (13). On the other hand, various Umbelliferous and Citrus (Rutaceae) plants are often used as traditional medicine in the form of crude drugs (14).

Using a microsomal system, furanocoumarins isolated 
from Umbelliferous crude drugs such as Baizhi (Angelica dahurica root), Hamaudo (Angelica japonica root), Qianghuo (Notopterygium incisum root), Fangfeng (Saposhnikovia divaricata root) and Yunnan Qianghuo (Pleurospermum rivulorum root) have been shown to inhibit CYP3A activity (15). The crude drugs are traditionally used as a hot water decoction or alcoholic infusion. Therefore, the evaluation of inhibitory potentials of the crude drugs in their therapeutic formulations is essential. In the present study, the decoction or infusion of the suspected crude drugs and related traditional prescriptions have been investigated for their CYP3A inhibition and the relationship of inhibitory potencies with their furanocoumarin compositions.

\section{MATERIALS AND METHODS}

\section{Chemicals}

Testosterone was purchased from Sigma Chemical Co. (St. Louis, MO, USA). $\beta$-NADP ${ }^{+}$, D-glucose 6-phosphate disodium salt and glucose-6-phosphate dehydrogenase were purchased from Oriental Yeast, Ltd. (Osaka).

The other reagents were of analytical grade from Wako Pure Chemical Industries, Ltd. (Osaka).

The standard furanocoumarin derivatives from Umbelliferous crude drugs verified in our previous study (15) were used to identify the furanocoumarin composition of crude drug samples.

Crude drugs and prescriptions of traditional herbal medicines

Several Umbelliferous or Citrus crude drugs, reported to contain furanocoumarins (14), were purchased from a local drug store (Sendai) or were kindly supplied by two distributors of medicinal materials (Nanjing, China). Voucher specimens are deposited at the Division of Drug Metabolism and Molecular Toxicology, Graduate School of Pharmaceutical Sciences, Tohoku University, Japan. Available information about these crude drugs is listed in Table 1.

Several prescriptions containing at least one of the suspected crude drugs were also investigated. A prescription of crude drugs (Table 2) was from the same Japanese drug store, whereas four commercially formulated prescriptions (Tables 3-6) were obtained at a Chinese dispensary.

\section{Microsomal testosterone $6 \beta$-hydroxylation}

Microsomal testosterone $6 \beta$-hydroxylation was used as an index of selective CYP3A activity in human livers. In brief, microsomes $(0.14 \mathrm{mg})$ centrifugally prepared from human liver samples (obtained from organ donors or patients undergoing liver resection) were incubated in a reaction mixture $(0.5 \mathrm{ml})$ containing an NADPH generating system and $0.2 \mathrm{mM}$ testosterone. To avoid interference from crude drugs, testosterone metabolites were separated on a Chemcopak column (Chemcosorb 5-ODS-H, 150 $\times 6 \mathrm{~mm}$ i.d.; Chemco Scientific Co., Ltd., Osaka), which was guarded with a precolumn (Nucleosil 120-5C $18,5 \mu \mathrm{M}$, $23 \times 4.0 \mathrm{~mm}$ i.d.; Chemco Scientific Co., Ltd.). A gradient of aqueous methanol ( $40 \%$ for $5 \mathrm{~min}, 40-48 \%$ over $10 \mathrm{~min}, 48-60 \%$ over $15 \mathrm{~min}, 60-70 \%$ over $15 \mathrm{~min}, 70 \%$ for $5 \mathrm{~min}, 70-40 \%$ over $5 \mathrm{~min}$ ) was used as the mobile phase. The other experimental details are the same as described previously (10).

Table 1. Available information of the crude drugs tested in this study

\begin{tabular}{|c|c|c|c|c|}
\hline Crude drug & $\begin{array}{c}\text { Dosage range } \\
\text { (g/day) }\end{array}$ & Lot & Source & Plant name \\
\hline \multirow[t]{3}{*}{ Baizhi/Byakushi } & $2-12$ & $\mathrm{BZ1}$ & Japan & $\begin{array}{l}\text { Angelica dahurica BENTH. et HOOK. var } \\
\text { dahurica BENTH. et HOOK }\end{array}$ \\
\hline & & BZ2 & Zhejiang, China & Angelica dahurica var. formosana \\
\hline & & BZ3 & Sichuan, China & Angelica dahurica \\
\hline \multirow[t]{2}{*}{ Qianghuo/Kyokatsu } & $2-9$ & QH1 & Qinghai, China & Notopterygium incisum or $N$. forbesii \\
\hline & & $\mathrm{QH} 2$ & Qinghai, China & Notopterygium incisum or $N$. forbesii \\
\hline Duhuo/Dokukatsu & $2-9$ & DH1 & Hubei, China & Angelica biserrata \\
\hline Fangfeng/Bofu & $3-9$ & FF1 & (Uncertain) $^{\mathrm{a}}$ & Saposhnikovia divaricata \\
\hline \multirow[t]{2}{*}{ Danggui/Toki } & $3-9$ & DG1 & $(\text { Uncertain })^{\mathrm{a}}$ & Angelica acutiloba \\
\hline & & DG2 & Gansu, China & Angelica sinensis \\
\hline \multirow[t]{2}{*}{ Zhishi/Kijitsu } & $2-9$ & $\mathrm{ZS} 1$ & $(\text { Uncertain })^{\mathrm{a}}$ & Citrus aurantium (immature fruit) \\
\hline & & $\mathrm{ZS} 2$ & Jiangxi, China & Citrus aurantium (immature fruit) \\
\hline Zhiqiao/Kikoku & $2-9$ & ZQ1 & Jiangxi, China & Citrus aurantium (fruit) \\
\hline
\end{tabular}

Crude drugs are listed as their common name in Chinese/Japanese. Dosage range is based on the Pharmacopoeia of P. R. China (2000 edition) and the Japanese Pharmacopoeia (13th edition). The underground parts (or as indicated separately) of a plant are dried for use. ${ }^{\text {a } O b t a i n e d ~ a t ~ a ~ d r u g ~ s t o r e ~(S e n d a i) ~ w i t h o u t ~ k n o w i n g ~ t h e ~ p l a c e ~ o f ~ p r o d u c t i o n . ~}$ 
Table 2. Prescription of Qing-shang-juan-tong-tang (Sejyo-kentsuto, SKT)

\begin{tabular}{lc}
\hline Crude drug (Latin name ${ }^{\mathrm{a}}$ ) & $\begin{array}{c}\text { Daily dose } \\
(\mathrm{g})\end{array}$ \\
\hline Maidong (Radix ophiopogonis) & 6.0 \\
Baizhi (Radix angelicae dahuricae, BZ1) & 3.0 \\
Huangqin (Radix scutellariae) & 5.0 \\
Manjingzi (Fructus viticis) & 2.0 \\
Qianghuo (Rhizoma et radix notopterygii) & 3.0 \\
Xixin (Herba asari) & 1.0 \\
Duhuo (Radix angelicae pubescentis) & 3.0 \\
Gancao (Radix glycyrrhizae) & 1.0 \\
Fangfeng (Radix saposhnikoviae, FF1) & 3.0 \\
Baizhu (Rhizoma atractylodis macrocephalae) & 3.0 \\
Juhua (Flos chrysanthemi) & 2.0 \\
Danggui (Radix angelicae sinensis, DG1) & 3.0 \\
Shengjiang (Rhizoma zingiberis recens) & 3.0 \\
Chuanxiong (Rhizoma) & 3.0 \\
\hline
\end{tabular}

${ }^{a}$ Mainly based on the Pharmacopoeia of P. R. China (2000 edition).

Table 3. Formulated prescription of Huoxue Zhitong capsules (HZC, $0.25 \mathrm{~g} /$ capsule, four capsules, t.i.d.)

\begin{tabular}{lc}
\hline Crude drug (Latin name ${ }^{\mathrm{a}}$ ) & $\begin{array}{c}\text { Composition } \\
(\%, \mathrm{w} / \mathrm{w})\end{array}$ \\
\hline Danggui (Radix angelicae sinensis) & 44.4 \\
Sanqi (Radix notoginseng) & 8.9 \\
Ruxiang (Olibanum) & 8.9 \\
Bingpian (Borneolum syntheticum) & 2.2 \\
Tubiechong (Eupolyphaga seu steleophaga) & 22.2 \\
Zirantong (Pyritum) & 13.3 \\
\hline
\end{tabular}

${ }^{\mathrm{a}}$ Mainly based on the Pharmacopoeia of P. R. China (2000 edition).

Table 4. Formulated prescription of strong Gastrodiae Eucommiae capsules (GEC, $0.2 \mathrm{~g} /$ capsule, $4-6$ capsules, b.i.d.)

\begin{tabular}{lc}
\hline Crude drug (Latin name ${ }^{\mathrm{a}}$ ) & $\begin{array}{c}\text { Composition } \\
(\%, \mathrm{w} / \mathrm{w})\end{array}$ \\
\hline Tianma (Rhizoma gastrodiae) & 9.6 \\
Duzhong (Cortex eucommiae) & 10.2 \\
Chuanniuxi (Radix cyathulae) & 7.1 \\
Danggui (Radix angelicae sinensis) & 12.0 \\
Qianghuo (Rhizoma et radix notopterygii) & 12.0 \\
Duhuo (Radix angelicae pubescentis) & 6.0 \\
Dihuang (Radix rehmanniae) & 19.3 \\
Hujisheng (Herba visci) & 7.1 \\
Zhicaowu (Radix aconiti kusnezoffii preparata) & 1.2 \\
Fuzi (Radix aconiti lateralis preparata) & 1.2 \\
Xuanshen (Radix scrophulariae) & 7.1 \\
Gaoben (Rhizoma ligustici) & 7.1 \\
\hline
\end{tabular}

${ }^{a}$ Mainly based on the Pharmacopoeia of P. R. China (2000 edition).
Table 5. Formulated prescription of Maren Wan pills (MWP, $9 \mathrm{~g}$ or 45 pills, q.d. or b.i.d.)

\begin{tabular}{lc}
\hline Crude drug (Latin name $\left.{ }^{\mathrm{a}}\right)$ & $\begin{array}{c}\text { Composition } \\
(\%, \mathrm{w} / \mathrm{w})\end{array}$ \\
\hline Huomaren (Fructus cannabis) & 20 \\
Kuxingren (Semen armeniacae amarum) & 10 \\
Dahuang (Radix et rhizoma rhei) & 20 \\
Zhishi (Fructus aurantii immaturus) & 20 \\
Houpo (Cortex magnoliae officinalis) & 10 \\
Baishao (Radix paeoniae alba) & 20 \\
\hline a Mainly based on the Pharmacopoeia of P. R. China (2000 edition)
\end{tabular}

${ }^{a}$ Mainly based on the Pharmacopoeia of P. R. China (2000 edition).

Table 6. Formulated prescription of Qian Bai Biyan Pian tablets (QBT, $0.4 \mathrm{~g} /$ tablet, 3 - 4 tablets, t.i.d.)

\begin{tabular}{lc}
\hline Crude drug (Latin name $\left.{ }^{\mathrm{a}}\right)$ & $\begin{array}{c}\text { Composition } \\
(\%, \mathrm{w} / \mathrm{w})\end{array}$ \\
\hline Qianliguang (Herba senecionis scandentis) & 76.2 \\
Juanbai (Herba selaginellae) & 12.7 \\
Qianghuo (Rhizoma et radix notopterygii) & 0.5 \\
Juemingzi (Semen cassiae) & 7.6 \\
Mahuang (Herba ephedrae) & 2.5 \\
Chuanxiong (Rhizoma ligustici chuanxiong) & 0.3 \\
Baizhi (Radix angelicae dahuricae) & 0.3 \\
\hline
\end{tabular}

${ }^{\text {a }}$ Mainly based on the Pharmacopoeia of P. R. China (2000 edition).

Inhibition of testosterone $6 \beta$-hydroxylation by crude drugs and relative prescriptions

Traditionally, a prescription is prepared by boiling in water $(500-600 \mathrm{ml}$ for a daily dosage) until the volume was reduced to a half of the original volume, and only the supernatant of the resulting 'soup' (decoction) was ingested. Another way is to immerse the crude drugs in liquor (35$45 \%$ ethanol) for a period of time and ingest the supernatant (infusion). Therefore, both decoctions and infusions of the collected crude drugs were prepared separately to test their CYP3A inhibition.

In most experiments, the crude drugs or related prescriptions were tested at a $2.5 \%$ level in the reaction mixture with their hot water decoctions (or $40 \%$ ethanol infusion). This is because in the same in vitro reaction mixture, $2.5 \%$ of various grapefruit juice samples showed clear CYP3A inhibition (10), a single cup of grapefruit juice (150$300 \mathrm{ml}$ ) can cause in vivo drug interaction (7), which is quite similar to the way that a crude drug (or a traditional prescription) of a daily dosage was boiled in water until the volume is reduced to $200-300 \mathrm{ml}$ for administration.

A sample of crude drug $(0.4 \mathrm{~g})$ was cut and boiled in water $(100 \mathrm{ml})$ for $30-40 \mathrm{~min}$ to half the volume to make a decoction $(50 \mathrm{ml})$, mimicking a typical way that a crude drug for a minimum daily dosage ( $2 \mathrm{~g}$, see Table 1$)$ is de- 
cocted with $500 \mathrm{ml}$ water. For a preparation of an infusion, a sample of crude drug $(0.35 \mathrm{~g})$ was ground and immersed in $40 \%$ ethanol $(35 \mathrm{ml}$ ) for 45 days (vortex mixed once each week).

An aliquot of supernatant (12.5 $\mu \mathrm{l}$ or as assigned) of a crude drug decoction or infusion was dried with a centrifugal concentrator (CC-105; Tomy Seiko Co., Ltd., Tokyo) and resuspended with the reaction mixture to test the influence on CYP3A activity. The inhibitory effect on testosterone $6 \beta$-hydroxylation was expressed as the percentage of the activity in comparison to the controls.

Daily dosage amounts of the prescription of crude drugs (Table 2) were freshly prepared as instructed: the crude drugs for a daily dosage were pooled and boiled in water $(600 \mathrm{ml})$ for $50-60 \mathrm{~min}$ until the volume was reduced to half $(300 \mathrm{ml})$. Assuming that orally administered drugs (usually with about $200 \mathrm{ml}$ water) are absorbed from the gastrointestinal tract (near $37^{\circ} \mathrm{C}$ ) within a period of time $(2-6 \mathrm{~h})$, samples (one fourth of a single dose) of the four formulated prescriptions of crude drugs (Tables $3-6$ ) were separately disintegrated by shaking in water $(50 \mathrm{ml})$ at $37^{\circ} \mathrm{C}$ for $4 \mathrm{~h}$. Aliquots $(12.5 \mu \mathrm{l}$ or as assigned $)$ of the supernatants of these prescriptions were also tested separately for their effects on microsomal testosterone $6 \beta$-hydroxylation.

Effects on testosterone $6 \beta$-hydroxylation by preincubation of crude drugs and related prescriptions

As an index of a mechanism-based inhibition, samples of crude drugs and their related prescriptions were separately preincubated for $15 \mathrm{~min}$ in the reaction mixture containing microsomes and the NADPH generating system before addition of testosterone.

Identification of furanocoumarins in a Japanese Baizhi (BZ1) and their contribution to the inhibition of CYP3A activity

The hydrophobic components of all the decoction or infusion samples were analyzed after the ether extraction and high performance liquid chromatographic (HPLC) separation. An aliquot of the decoction or infusion of a crude drug or its related prescription was extracted three times with 1.6-fold volumes of diethyl ether. The ether layer was separated after vigorous vortex-mixing for $3 \mathrm{~min}$ and centrifugation at $5000 \mathrm{rpm}$ for $1 \mathrm{~min}$ and then evaporated in a centrifugal concentrator. An aliquot of residues dissolved in methanol was injected for HPLC analysis. The HPLC separation was conducted at room temperature with a YMC column (YMC-Pack ODS-A, $150 \times 4.6 \mathrm{~mm}$ i.d., S-5 $\mu \mathrm{m}$, 120A; YMC Co., Ltd., Kyoto) that was guarded with a filter. A gradient of aqueous methanol (30\% to 50\% over $20 \mathrm{~min} ; 50 \%$ to $85 \%$ over $15 \mathrm{~min} ; 100 \%$ for $5 \mathrm{~min}$ ) was used as the mobile phase, the flow rate was set at $1 \mathrm{ml}$ /min. The eluate was monitored by the absorption at 240 $\mathrm{nm}$. Authentic furanocoumarins were also injected to confirm the identities of peaks that appeared when a crude drug sample was injected.

For the infusion of BZ1, its HPLC fractions (in a continuous 5-min interval) or isolated main peaks were tested separately, each at the concentration level equivalent to that of the original infusion $(2.5 \%)$. The experimental details were as follows: $12.5 \mu \mathrm{l}$ of the infusion was tested in $0.5 \mathrm{ml}$ of reaction mixture (2.5\% of infusion); when $1.0 \mathrm{ml}$ of infusion was extracted and the resulted extracts was redissolved in $0.1 \mathrm{ml}$ of methanol, $1.25 \mu \mathrm{l}$ was tested for inhibition (2.5\% of extract) and $40 \mu \mathrm{l}$ was injected for HPLC separation; after HPLC separation, $156.25 \mu \mathrm{l}$ of the $5.0 \mathrm{ml}$-eluate fractions were tested for inhibition separately or after pooling all fractions (2.5\% of fraction); when a HPLC peak had been isolated from the eluate, it was dissolved in methanol and the concentration was adjusted until a $40 \mu$ l-injection showed the same peak height as that of the methanolic solution of infusion extract, then $1.25 \mu \mathrm{l}$ of such a peak solution was tested for inhibition (2.5\% of peak). Main HPLC peaks were identified by their UV absorption spectra and co-chromatography with authentic furanocoumarins.

To understand the influence of preparation (decoction or infusion) on the inhibitory effect of a crude drug, BZ1 was ground and divided into equal aliquots to prepare a decoction and an infusion. The infusion was prepared by shaking the ground $\mathrm{BZ} 1$ in $40 \%$ ethanol at $45^{\circ} \mathrm{C}$ for $24 \mathrm{~h}$ in this experiment. The residue of $\mathrm{BZ} 1$ decoction was infused with $40 \%$ ethanol at $45^{\circ} \mathrm{C}$ for $24 \mathrm{~h}$ and its CYP3A inhibition was also tested together with the decoction and direct infusion. Each preparation was tested with a 2.5-fold diluting series, and the concentration of inhibitor causing $50 \%$ reduction in activity relative to the control $\left(\mathrm{IC}_{50}\right)$ was determined graphically at the range when it showed concentration-depending inhibition. All three preparations were analyzed in parallel to compare their furanocoumarin compositions after ether extraction and HPLC separation.

\section{RESULTS}

\section{Inhibition of microsomal testosterone $6 \beta$-hydroxylation by crude drugs}

Inhibition of microsomal CYP3A activity was tested by the addition of a decoction or infusion of crude drugs at a $2.5 \%(\mathrm{v} / \mathrm{v})$ level in the reaction mixture (Fig. 1). For the decoctions, only one sample of Baizhi (BZ1) and one sample of Qianghuo (QH1) decreased the CYP3A activity to less than $50 \%$ of the control, the other samples added showed just marginal or no inhibitory effect. For the infusions, all the samples of Baizhi (BZ1 - 3) and Qianghuo (QH1 and 2) lowered the CYP3A activity to less than 30\% of the control. The other samples failed to decrease the CYP3A activity to less than $60 \%$ of the control, and two 


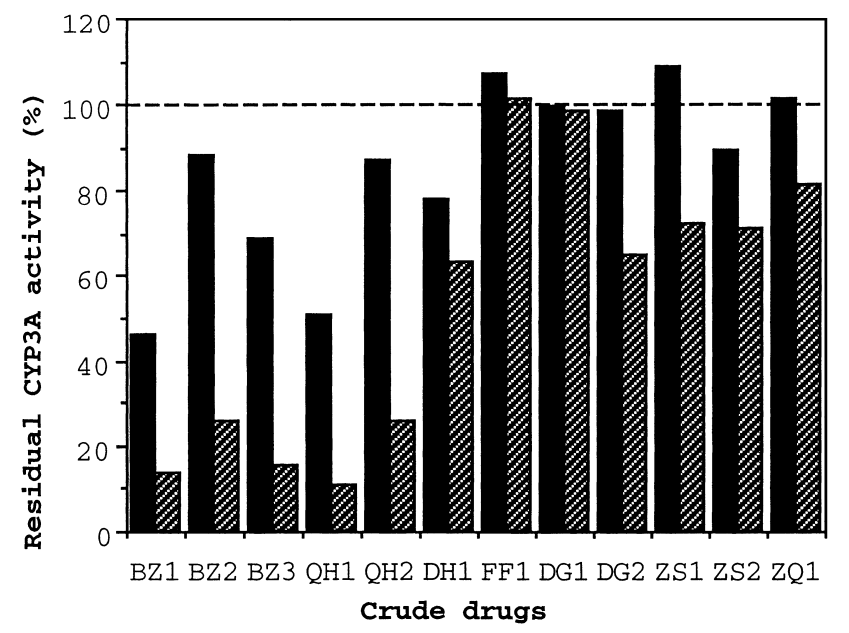

Fig. 1. Inhibition of microsomal CYP3A activity by crude drugs. See Table 1 for descriptions of the coded crude drugs. Each one was tested as its hot water decoction (filled column) or $40 \%$ ethanol infusion (hatched column) at the final concentration of $2.5 \%(\mathrm{v} / \mathrm{v})$. Residual CYP3A activity is the residual percentage of testosterone $6 \beta$-hydroxylation in human liver microsomes. See Materials and Methods for experimental details. Data are shown as the mean of at least duplicated determinations with variation under $10 \%$.

samples Fangfeng (FF1) and Danggui (DG1) showed no inhibition. In general, the infusion of a crude drug showed stronger inhibition than did the decoction of the same crude drug.

Components in each crude drug decoction or infusion were analyzed after ether extraction and HPLC separation. The sample with more or higher HPLC peaks tended to have stronger inhibition of CYP3A activity. The HPLC peaks were checked by comparison of their retention times with those of known furanocoumarins.

\section{Contribution of furanocoumarin components in the inhibi- tion of CYP $3 A$ by BZ1}

A sample of BZ1 showed the most abundant HPLC peaks, a typical HPLC chart of BZ1 infusion is shown in Fig. 2A. The HPLC eluate of BZ1 infusion was fractionally collected and tested at the same quantity corresponding to the original infusion. Fractions of $35-40 \mathrm{~min}$ (Fr8) and 45-60 min (Fr10, Fr11 and Fr12) showed clear inhibition of CYP3A activity, and the pooled aliquots of all fractions showed inhibitory potency equivalent to the original infusion or its ether extract (Fig. 2B). As shown in Fig. 2C each of the main peaks, separately isolated, showed inhibition equivalent to its corresponding fraction.

On the basis of previous reports $(16-21)$, UV absorption and HPLC co-chromatography, Peaks $1-8$ from BZ1 infusion were tentatively identified to be furanocoumarin derivatives as listed in Fig. 3. The $\mathrm{IC}_{50}$ values are data from a previous report (15).
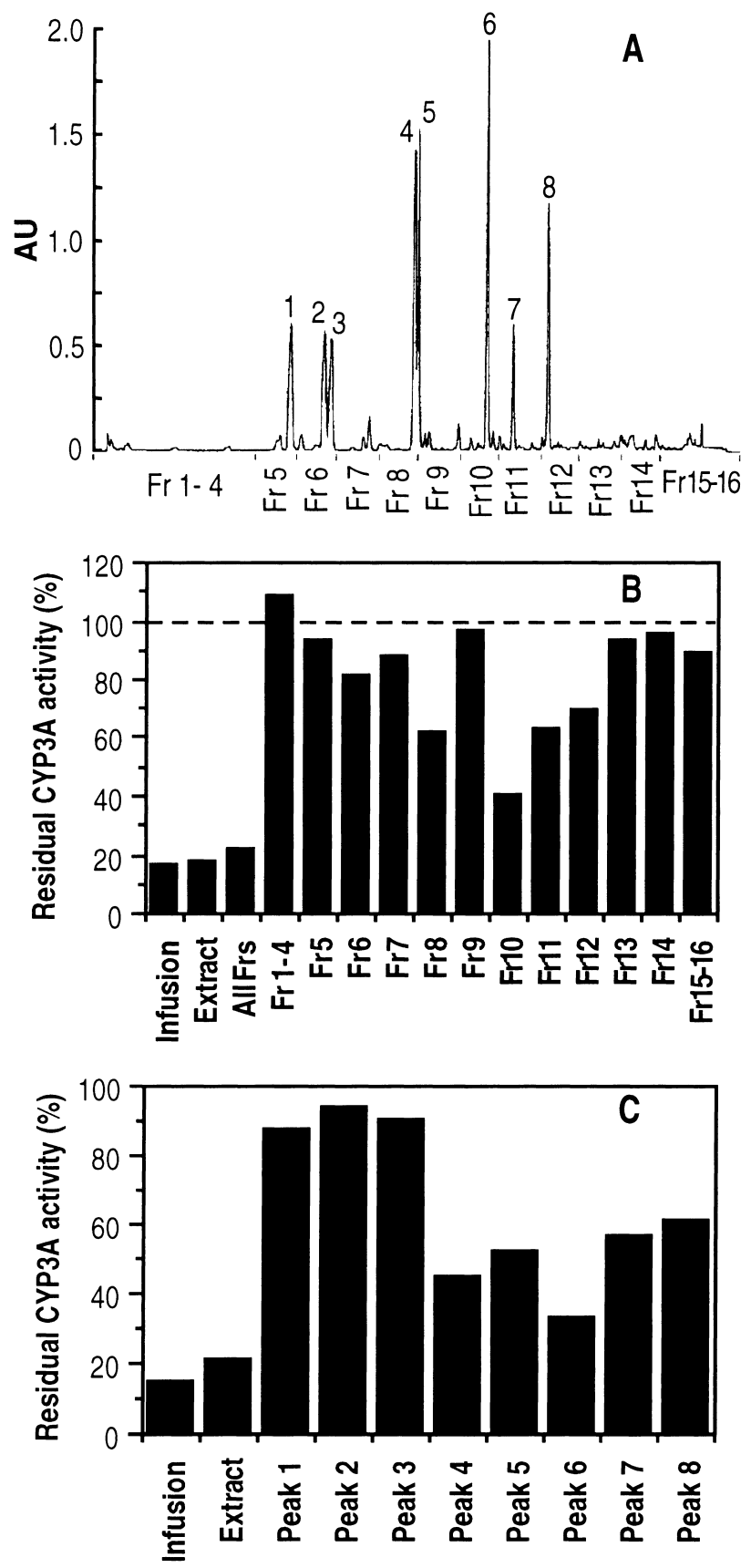

Fig. 2. HPLC chromatogram (A) of a Japanese Baizhi (BZ1) and CYP3A inhibition by its HPLC fractions (B) or main peaks (C). See Table 1 for a sample description of BZ1. Infusion: $40 \%$ ethanol extract of BZ1; extract: diethyl ether extract of the $40 \%$ ethanol infusion; $\operatorname{Fr}(\mathrm{s})$ : HPLC fraction(s) of the injected diethyl ether extract, collected separately at a 5-min interval and pooled as indicated; peak: isolated HPLC peak as numbered in the chromatogram; residual CYP3A activity: the residual percentage of testosterone $6 \beta$-hydroxylation in human liver microsomes. See Materials and Methods and Results for experimental details. All data in panels B and C are means of at least duplicated determinations with variation under $10 \%$.

The compositions of furanocoumarins are quite different among BZ1 decoction, infusion of decoction residuals and 


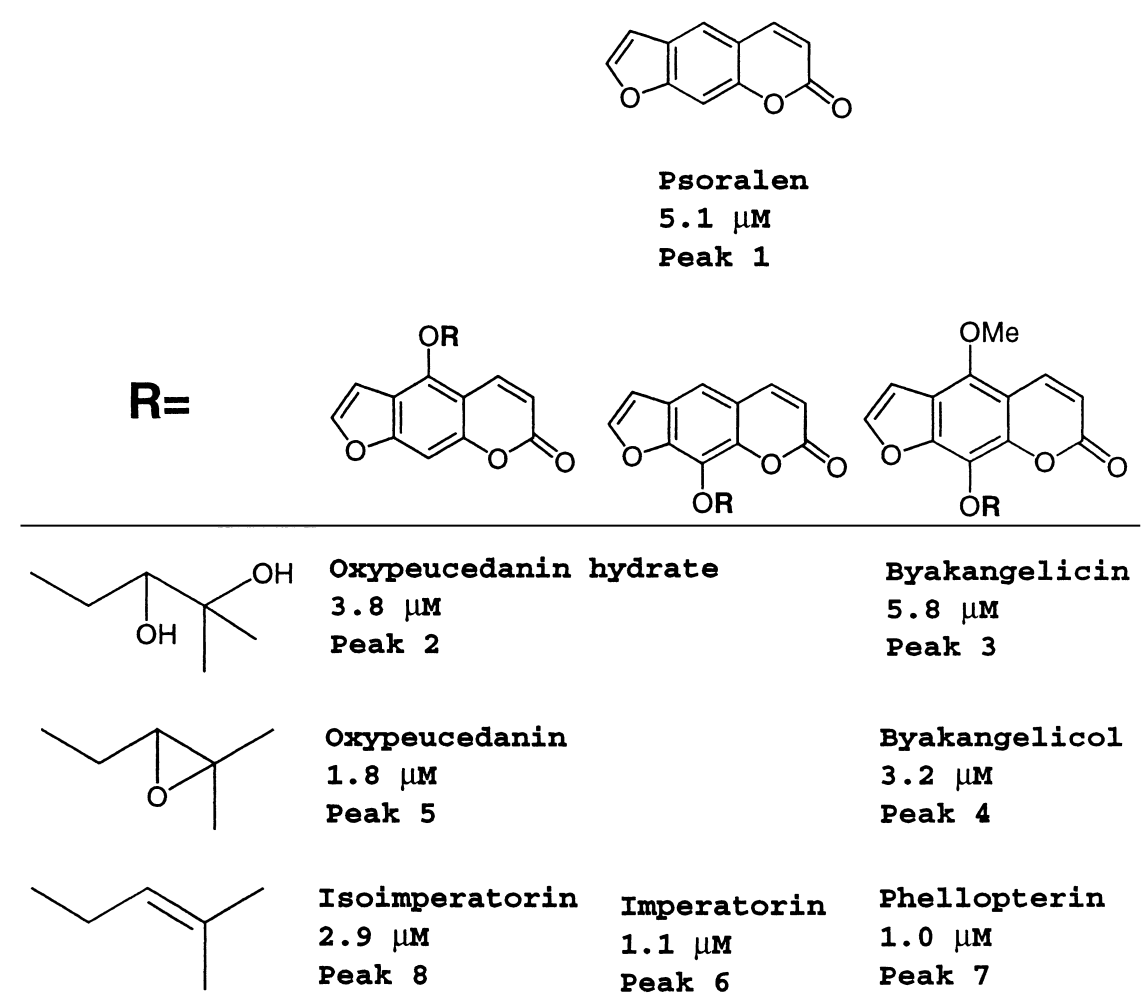

Fig. 3. Furanocoumarin structures and their inhibitory potencies on CYP3A activity. The order of listing for each compound is as follows: chemical name, $\mathrm{IC}_{50}$ value on testosterone $6 \beta$-hydroxylation in human liver microsomes and correspondence to the peak in the chromatogram of Fig. $2 \mathrm{~A}$.

direct infusion (Fig. 4). The decoction showed peaks $2-5$ as main components, whereas the direct infusion showed much higher contents of peak $4-8$. Peak 8 was not detected in the decoction, but appeared in the infusion of the residue of decoction. The content was, however, much lower than that of direct infusion, which contained more numbers and higher contents of furanocoumarins. In addition, preincubation prior to addition of a test probe, testosterone, resulted in the higher inhibition of the microsomal CYP3A activity (Table 7).

Inhibition of microsomal testosterone $6 \beta$-hydroxylation by prescriptions of crude drugs

At a $2.5 \%(\mathrm{v} / \mathrm{v})$ level, the decoction of Sejyo-kentsu-to (SKT) (Table 2) showed 67\% inhibition of the CYP3A activity without preincubation, and the extent of the inhibition increased to $78 \%$ after preincubation (Fig. 5). For the separately prepared decoctions of crude drugs (Table 1), some also showed increased inhibition after preincubation (Fig. 5).

None of the water disintegrated samples of the four formulated prescriptions (Tables 3-6) showed appreciable inhibition at the $2.5 \%$ or $7.5 \%(\mathrm{v} / \mathrm{v})$ level. At a much higher level of $25 \%(\mathrm{v} / \mathrm{v})$, the sample of Huoxue Zhitong capsules (HZC) (Table 3), Gastrodiae Eucommiae capsules (GEC)
(Table 4), Maren Wan pills (MWP) (Table 5) and Qian Bai Biyan Pian tablets (QBT) (Table 6) inhibited 53\%, 22\%, $77 \%$ and $80 \%$ of the CYP3A activity, respectively (Fig. 6). When the ether extracts and water phase residuals of these samples were tested in parallel, the ether extracts of HZC and GEC showed similar potencies of inhibition to their own original preparations. In contrast, the water phase residue of MWP showed similar potency of inhibition to the ether extract and both the preparations showed weaker potencies than that of the origin. The water phase residue of QBT, however, showed a stronger inhibitory effect than the ether extract.

\section{DISCUSSION}

The present study was triggered by the discovery that specific furanocoumarins were the causative components in grapefruit juice-drug interaction $(8-11)$; the selections of crude drugs are based on the reason that they have been confirmed to contain furanocoumarins. The selection focused on the crude drugs that are used as traditional medicine in China and/or Japan, and only those widely used were tested in considering clinical significance (14). Also for clinical reasons, the hot water decoction or $40 \%$ ethanol infusion of a crude drug, not the crude drug itself, was 

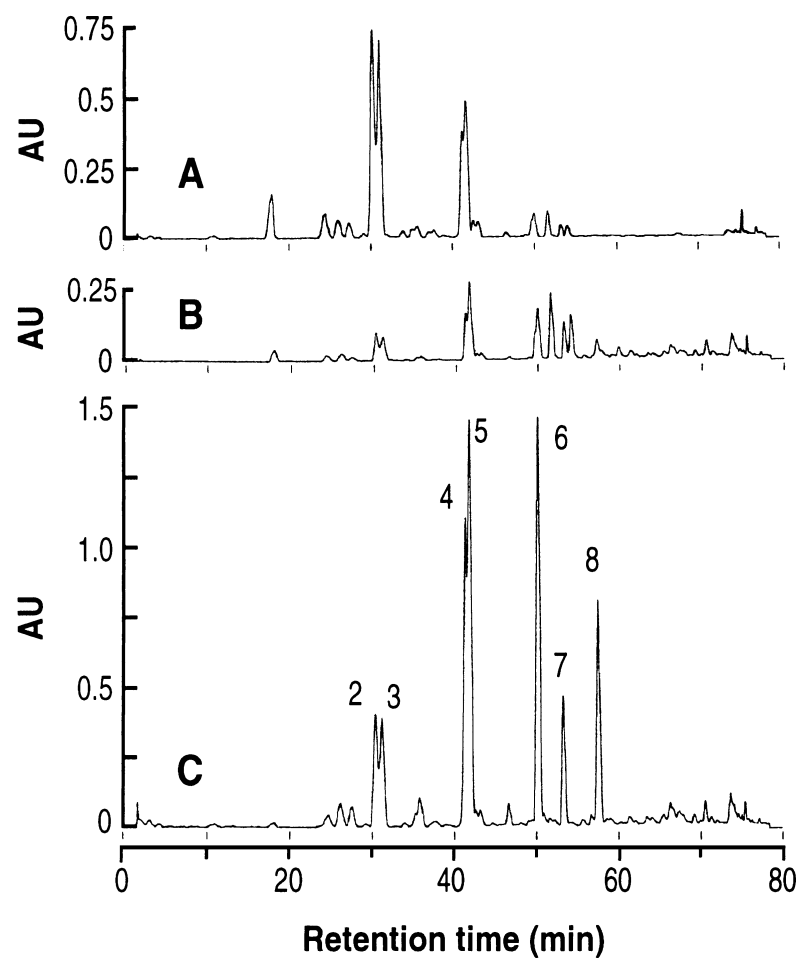

Fig. 4. Component differences among decoction (A), infusion post decoction (B) and direct infusion (C) of a Japanese Baizhi (BZ1). See Table 1 for information about BZ1; the numbered peaks are the same as in Figs. 2 and 3. See Materials and Methods for experimental details.

Table 7. $\mathrm{IC}_{50}$ values of a Japanese Baizhi (BZ1) on microsomal CYP3A activity

\begin{tabular}{lcc}
\hline & \multicolumn{2}{c}{$\mathrm{IC}_{50}(\mu \mathrm{g} / \mathrm{ml})$} \\
\cline { 2 - 3 } & $\begin{array}{c}\text { Without } \\
\text { preincubation }\end{array}$ & $\begin{array}{c}\text { With } \\
\text { preincubation }\end{array}$ \\
\hline Decoction $^{\mathrm{a}}$ & 110 & 34 \\
Infusion of the decoction residues $^{\mathrm{b}}$ & 84 & 17 \\
Infusion $^{\mathrm{c}}$ & 30 & 9
\end{tabular}

See Table 1 for information on BZ1; CYP3A activity was assessed with testosterone $6 \beta$-hydroxylation by human liver microsomes; see Materials and Methods for experimental details. $\mathrm{IC}_{50}$ values were estimated graphically, corresponding to the original quantity of BZ1 used for preparation of the decoction or infusion. ${ }^{\text {aSupernatant after }}$ extraction with hot water of ground BZ1. 'Supernatant after extraction with $40 \%$ ethanol of the precipitate of decoction. 'Supernatant after direct extraction with $40 \%$ ethanol of ground BZ1.

checked for its CYP3A inhibition and its furanocoumarin composition, because the decoction or infusion is more commonly used for ingestion.

Although pharmacognostic books or original reports indicated that furanocoumarins are contained in the selected herbs (Table 1), the samples checked in this study are not

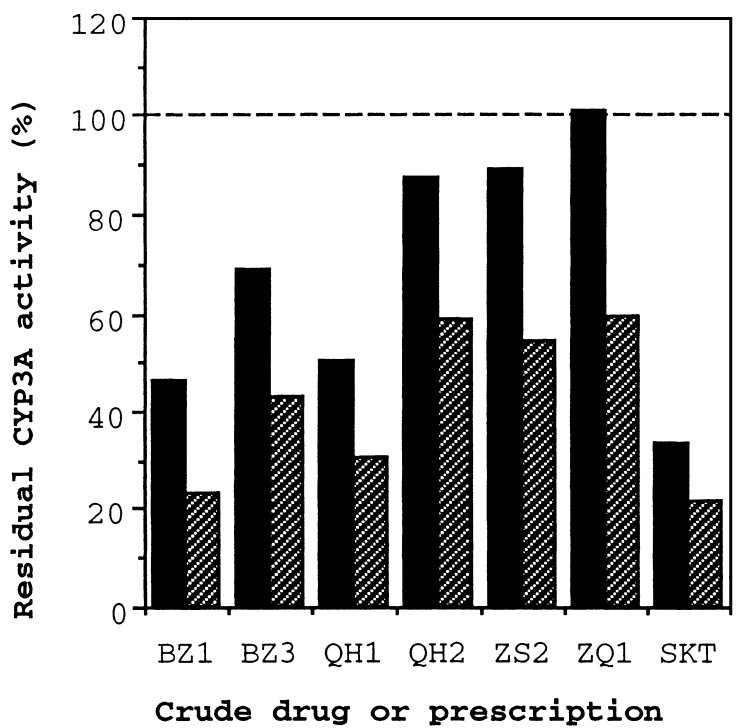

Fig. 5. Inhibition of CYP3A activity by the decoctions of crude drugs and relative prescriptions with (hatched column) or without (filled column) preincubation. See Table 1 for information on the crude drugs BZ1, BZ3, QH1, QH2, ZS2 and ZQ1. See Table 2 for information on the prescriptions SKT. All the samples were tested, respectively, as their decoctions at the level of $2.5 \%(\mathrm{v} / \mathrm{v})$ in the reaction mixture. Residual CYP3A activity is shown as the residual percentage of testosterone $6 \beta$-hydroxylation in human liver microsomes. See Materials and Methods for experimental details. Data are shown as the mean of at least duplicated determinations with variation under $10 \%$.

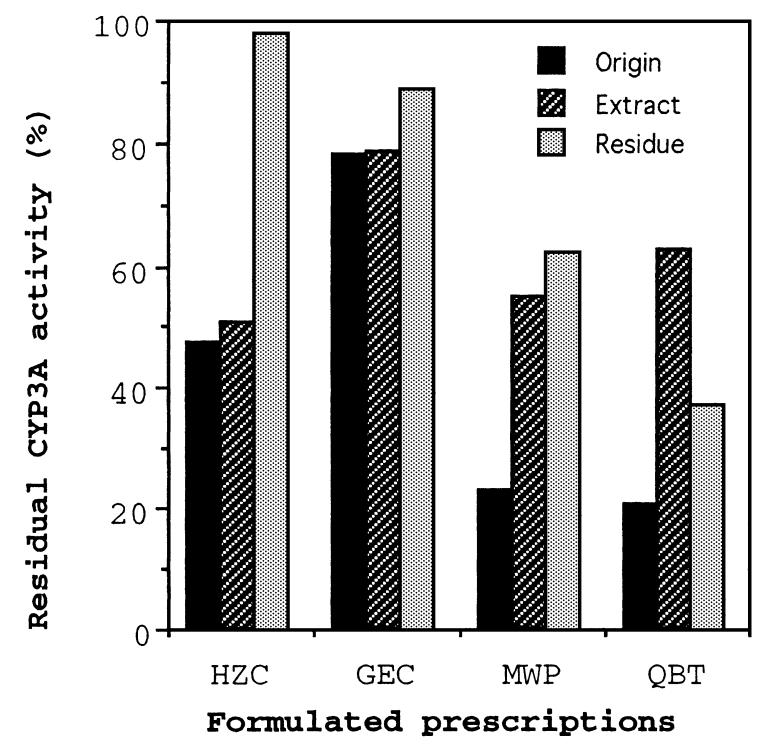

Fig. 6. Inhibition of CYP3A activity by the formulated prescriptions (Origin) and by their ether extractable (Extract) and unextractable (Residue) components. See Tables $3-6$ for information on the formulated prescriptions HZC, GEC, MWP and QBT respectively. The preparations were disintegrated in water and then tested directly or after ether extraction, at the level of $25 \%(\mathrm{v} / \mathrm{v})$ in the reaction mixture. Residual CYP3A activity is the residual percentage of testosterone $6 \beta$-hydroxylation in human liver microsomes. See Materials and Methods for experimental details. Data are shown as the mean of at least duplicated determinations with variation under $10 \%$. 
consistently showing furanocoumarin existence in the form of decoction or infusion. This is partly because the difference in places of production or species of plant $(20,22-$ $25)$. On the other hand, the co-variations of furanocoumarin amounts among the samples of crude drugs with their potencies of CYP3A inhibition (Fig. 1) suggest that furanocoumarins did play the major role in these crude drugs for their inhibitory effects on a drug metabolizing enzyme. Danggui is contained in more than 70 traditional prescriptions and has been noted in some books to contain furanocoumarins such as bergapten, xanthotoxin and isopimpinellin. As shown in Fig. 1, only the infusion of DG2 inhibited CYP3A activity marginally in the two samples tested (DG1 from Angelica acutiloba and DG2 from Angelica sinensis). These data are consistent with the appearance of an appreciable HPLC peak corresponding to imperatorin.

Fangfeng is contained in around 25 traditional prescriptions and has been reported to contain furanocoumarins such as psoralen, bergapten, xanthotoxin, imperatorin and phellopterin (25). The tested sample (FF1 from Saposhnikovia divaricata), however, showed neither appreciable CYP3A inhibition (Fig. 1) nor possible furanocoumarin peaks in HPLC.

Qianghuo is used in around 13 traditional prescriptions. In addition to the main components isoimperatorin and notopterol, up to 15 furanocoumarin derivatives have been found in this crude drug with varying composition and/or content among samples from different sources $(22-24$, 26). For the two samples $(\mathrm{QH} 1$ and $\mathrm{QH} 2$, both from Notopterygium incisum or N.forbesii), the decoction of QH1 showed appreciable peaks corresponding to isoimperatorin and notopterol, and these peaks were much higher in the infusion of this sample; For QH2, its decoction showed trace amounts of a detectable peak corresponding to notopterol, but its infusion showed quite a high peak corresponding to notopterol and some other appreciable peaks among which one corresponded to isoimperatorin. The samples of QH1 and QH2 showed variable extents of CYP3A inhibition (Fig. 1), but the extents were in good agreement with their furanocoumarin contents.

Duhuo is a crude drug closely related to Qianghuo (22). It appears in around six traditional prescriptions and has been shown to contain furanocoumarins such as bergapten, byakangelicin, isoimperatorin, psoralen and xanthotoxin $(22,27)$. The sample we obtained (DH1 from Angelica biserrata) showed around ten detectable peaks in the HPLC chromatogram. Although the peak corresponding to isoimperatorin was higher in the infusion than that in the decoction, the rest of the peaks (unidentified but more hydrophilic) were higher in the decoction than in the infusion. Both decoction and infusion of DH1 showed weak CYP3A inhibition and the potency of infusion was comparably stronger.
Baizhi is used in around 15 traditional prescriptions, and up to 18 furanocoumarin derivatives have been isolated from various samples of this crude drug $(16-21)$. Among the samples we collected (BZ1 from Angelica dahurica BENTH. et HOOK. var. dahurica BENTH. et HOOK, BZ2 from Angelica dahurica var. formosana and BZ3 from Angelica dahurica), the clear relationship between the CYP3A inhibition and the furanocoumarin abundance were obtained both with the infusion and decoction of BZ1 (Figs. $2-4$ and Table 7); for BZ2, peaks 2 (oxypeucedanin hydrate) and 3 (byakangelicin) were detectable and peaks 6 (imperatorin), 7 (phellopterin) and 8 (isoimperatorin) were at appreciable levels in its infusion, and the same peaks were found in its decoction but at very low levels; for BZ3, its infusion showed a detectable level of peak 3, appreciable levels of peaks 5 (oxypeucedanin), 7 and 8 , and quite a high level of peak 6 , and the same pattern appeared in its decoction but at much lower levels with all peaks. Similar to BZ1, BZ2 and BZ3 also showed CYP3A inhibition (Fig. 1) with the potencies for their infusions and decoctions in correspondence to their furanocoumarins compositions.

Based on the results of this study on the umbelliferous crude drugs (Fig. 1), we suggest that special attention should be paid to those prescriptions that contain Baizhi or Qianghuo because these two crude drugs tend to contain more species and higher contents of furanocoumarins. The samples of a crude drug under the same label (trade name) from different sources sometimes showed quite different potencies of CYP3A inhibition. Therefore, the present result do not exclude the possibility that the crude drugs exhibiting negligible inhibitory effects in this study may have strong CYP3A inhibition in samples from different sources.

Zhishi and Zhiqiao are usually dried fruits of a sour orange (Citrus aurantium), which is a species close to grapefruit by botanical taxonomy (28), and they can be found in about 30 traditional prescriptions. Some books mentioned that it contains imperatorin and isoimperatorin. 6',7'-Dihydroxybergamottin (DHB, a CYP3A inhibitor in grapefruit juice) was also found in this crude drug (29). For the three samples we collected (ZS1 from Japan and ZS2 and ZQ1 from the same place of production in China), ZS1 showed almost no HPLC peak in its decoction, but showed a detectable peak corresponding to isoimperatorin in its infusion; both decoctions of ZS2 and ZQ1 contain detectable levels of peaks including two corresponding to imperatorin and isoimperatorin, and all the peaks appeared at higher levels in their infusions. Again, the low levels of furanocoumarins in the infusions could be the causes of their marginal CYP3A inhibition (Fig. 1).

Furanocoumarins from grapefruits have been proved to be at least partially mechanism-based inhibitors $(10,30)$, so it is also necessary to check if the crude drugs containing furanocoumarins could cause mechanism-based inhibition. 
After preincubation, both the decoction and infusion of BZ1 showed over threefold stronger inhibition of CYP3A activity (Table 7). Their furanocoumarin compositions were, however, different quantitatively (Fig. 4), it is difficult to identify a specific furanocoumarin derivative that plays a major role in the mechanism-based inhibition. When the decoctions of all crude drug samples were tested at the $2.5 \%(\mathrm{v} / \mathrm{v})$ level, only those of BZ1, BZ3, QH1, $\mathrm{QH} 2, \mathrm{ZS} 2$ and ZQ1 showed stronger inhibition after preincubation (Fig. 5). The other samples of crude drugs in Table 1 were also tested, but they showed no difference after preincubation (data not shown), and consistently, they contained no or very low level of furanocoumarin. An interesting phenomenon was observed with the samples of QH2, ZS2 and ZQ1. These samples showed very weak or even no inhibition without preincubation, but showed clear preincubatory effects. One possibility is that these samples may contain furanocoumarin dimers at quite low levels, because in our previous studies, furanocoumarin dimers had shown much stronger mechanism-based inhibition in comparison with monomers $(15,30)$, but the contents of dimers were much lower than those of monomers in grapefruit juice (close to the plant of ZS and ZQ) (10).

In most cases, a traditional prescription is composed of multiple crude drugs to achieve the best therapeutic effects, so it is also necessary to check if a suspected crude drug will show inhibition on drug metabolism enzymes when it is contained in a traditional prescription and processed together with other crude drugs for administration. The crude drugs Baizhi, Fangfeng and Danggui contained in prescription SKT (recipe shown in Table 2) should be the same as BZ1, FF1 and DG1, respectively, because the crude drugs and the prescriptions were bought in the same local drug store of traditional medicines. Since none of the decoctions of FF1 and DG1 showed CYP3A inhibition at comparable concentration level when tested separately (Fig. 1), it is reasonable that the decoction of SKT showed CYP3A inhibition similar to that when the decoction of BZ1 was tested separately (Fig. 5). HPLC analysis of the decoction of SKT also showed a chromatogram quite similar to that of the decoction of BZ1 (Fig. 4A), both showing oxypeucedanin hydrate (Peak 2) and byakangelicin (Peak 3) as main components at comparable levels. (Qianhuo was contained in prescription SKT, but this sample was found to be a local substitute from a different plant, and its separately prepared decoction showed no CYP3A inhibition or furanocoumarin existence; data not shown.)

For the separately tested Umbelliferous or Citrus crude drugs (Table 1) and a freshly prepared prescription containing the suspected crude drugs (Table 2), it can be summarized that their inhibitory potencies on CYP3A activity were mainly dependent on their hydrophobic components, especially on those identified as furanocoumarins. But for the samples of the formulated prescriptions (Tables 3-6), the present results (Fig. 6) implied that some hydrophilic components, in stead of the hydrophobic furanocoumarins, might also inhibit CYP3A activity, especially in the case of MWP or QBT.

The samples of the formulated prescriptions showed no detectable HPLC peaks corresponding to possible furanocoumarins; this might be because of the way we prepared the samples. The results of BZ1 showed that furanocoumarins appeared less in the hot water decoction (Fig. 4A) than in the $40 \%$ ethanol infusion (Fig. 4C); since we just put the formulated prescriptions in water and shook it at $37^{\circ} \mathrm{C}$, it is predictable that furanocoumarins (if they existed) will appear even less. Since these formulated prescriptions are made for direct administration but the details of process are not clear, the possible contribution of furanocoumarins (if they remain in the resulted products) on CYP3A inhibition can not be excluded at the present stage.

In conclusion, in vitro investigations in this study showed that crude drugs containing furanocoumarins or relative traditional prescriptions could inhibit CYP3A activity similar to grapefruit juice, which indicates that they may also cause in vivo drug interactions, and mechanismbased inhibition is sometimes involved. On the other hand, since we have found that furanocoumarin could also inhibit other CYP subtypes (30), it is possible that the inhibitory effects of the crude drugs containing furanocoumarins may be not limited to those drugs that are CYP3A substrates.

\section{Acknowledgment}

This study was supported in part by the Rotary Yoneyama Memorial Foundation (to L.-Q. Guo) and by a grant from the Ministry of Health, Labour and Welfare, Japan.

\section{REFERENCES}

1 De Smet PA: Health risks of herbal remedies. Drug Saf 13, $81-$ 93 (1995)

2 Nishimura N, Naora K, Hirano H and Iwamoto K: A Chinese traditional medicine, sho-saiko-to (xiao-chaihu-tang), reduces the bioavailability of tolbutamide after oral administration in rats. Am J Chin Med 27, 355 - 363 (1999)

3 Ohnishi N, Yonekawa Y, Nakasako S, Nagasawa K, Yokoyama T, Yoshioka M and Kuroda K: Studies on interactions between traditional herbal and Western medicines. I. Effects of Shoseiryu-to on the pharmacokinetics of carbamazepine in rats. Biol Pharm Bull 22, 527 - 531 (1999)

4 Koizumi K, Iijima K, Nohmi M, Nakayama S and Oguchi K: Effects of byakushi and ogon on the hepatic drug metabolizing enzymes in rats. Folia Pharmacol Jpn (Nippon Yakurigaku Zasshi) 104, 413 - 419 (text in Japanese with English abstract) (1994)

5 Mayanagi M, Nakayama S and Oguchi K: Effects of SinoJapanese herbs in the family Umbelliferae on the hepatic drug metabolizing enzymes and lipid peroxidation in rats. Folia Pharmcacol Jpn (Nippon Yakurigaku Zasshi) 99, 115-121 
(1992) (text in Japanese with English abstract)

6 Ishihara K, Kushida H, Yuzurihara M, Wakui Y, Yanagisawa T, Kamei H, Ohmori S and Kitada M: Interaction of drugs and Chinese herbs: Pharmacokinetic changes of tolbutamide and diazepam caused by extract of Angelica dahurica. J Pharm Pharmacol 52, 1023 - 1029 (2000)

7 Bailey DG, Malcolm J, Arnold O and Spence JD: Grapefruit juice-drug interactions. Br J Clin Pharmacol 46, 101-110 (1998)

8 Edwards DJ, Bellevue FH 3rd and Woster PM: Identification of 6',7'-dihydroxybergamottin, a cytochrome $\mathrm{P} 450$ inhibitor, in grapefruit juice. Drug Metab Dispos 24, 1287 - 1290 (1996)

9 Fukuda K, Ohta T, Oshima Y, Ohashi N, Yoshikawa M and Yamazoe Y: Specific CYP3A4 inhibitors in grapefruit juice: furocoumarin dimers as components of drug interaction. Pharmacogenetics 7, 391 - 396 (1997)

10 Guo LQ, Fukuda K, Ohta T and Yamazoe Y: Role of furanocoumarin derivatives on grapefruit juice-mediated inhibition of human CYP3A activity. Drug Metab Dispos 28, 766-771 (2000)

11 He K, Iyer KR, Hayes RN, Sinz MW, Woolf TF and Hollenberg PF: Inactivation of cytochrome P450 3A4 by bergamottin, a component of grapefruit juice. Chem Res Toxicol 11, 252-259 (1998)

12 Pathak MA, Daniels F and Fitzpatrick TB: The presently known distribution of furocoumarins (psoralens) in plants. J Invest Dermatol 39, 225 - 239 (1962)

13 Pathak MA and Fitzpatrick TB: The evolution of photochemotherapy with psoralens and UVA (PUVA): 2000 BC to 1992 AD. J Photochem Photobiol B Biol 14, 3 - 22 (1992)

14 Namba T: Coloured Illustrations of Wakan-Yaku (The Crude Drugs in Japan, China and the Neighbouring Countries), Hoikusha Publishing Co, Ltd, Osaka (1980) (in Japanese with multiple indexes in English)

15 Guo LQ, Taniguchi M, Xiao YQ, Baba K, Ohta T and Yamazoe $Y$ : Inhibitory effect of natural furanocoumarins on human microsomal cytochrome P450 3A activity. Jpn J Pharmacol 82, $122-129(2000)$

16 Kwon YS, Kobayashi A, Kajiyama S, Kawazu K, Kanzaki H and Kim CM: Antimicrobial constituents of Angelica dahurica roots. Phytochemistry 44, $887-889$ (1997)

17 Bergendorff O, Dekermendjian K, Nielsen M, Shan R, Witt R, Ai $J$ and Sterner O: Furanocoumarins with affinity to brain benzodiazepine receptors in vitro. Phytochemistry 44, 1121 1124 (1997)

18 Kozawa M, Baba K, Okuda K, Fukumoto T and Hata K: Studies on chemical components of "Bai Zhi" (Supplement 1) on coumarins from "Japanese Bai Zhi". Shoyakugaku Zasshi 35, 90 - 95 (1981) (text in Japanese with English abstract)

19 Hata K, Kozawa M and Yen KY: Pharmacognostical studies on Umbelliferous plants. XIX. Studies on Chinese drug "Byaku-shi". (4). On the coumarins of the roots of Angelica dahurica Benth. et Hook. var. dahurica and A. dahurica Benth. et Hook. var. pai-chi Kimura, Hata et Yen. Yakugaku Zasshi 83, $606-610$ (1963) (text in Japanese with English abstract)

20 Hata K, Kozawa M, Yen KY and Kimura Y: Pharmacognostical studies on Umbelliferous plants. XX. Studies on Chinese drug "Byaku-shi". (5). On the coumarins of the roots of Angelica formosana Boiss. and A. anomala Lall. Yakugaku Zasshi 83, $611-614$ (1963) (text in Japanese with English abstract)

21 Kimura Y, Okuda $\mathrm{H}$ and Baba K: Histamine-release effectors from Angelica Dahurica var. dahurica root. J Nat Prod 60, 249 - 251 (1997)

22 Kozawa M and Tanaka O: Chemical Constituents of Du-huo and Qiang-huo. Gendai Toyo Igaku 8, 69 - 73 (1987) (in Japanese)

23 Zschocke S, Lehner $\mathrm{M}$ and Bauer R: 5-Lipoxygenase and cyclooxygenase inhibitory active constituents from Qianghuo (Notopterygium incisum). Planta Med 63, 203 - 206 (1997)

$24 \mathrm{Gu}$ ZM, Zhang DX, Yang XW, Hattori $M$ and Namba T: Isolation of two new coumarin glycosides from Notopterygium forbesii and evaluation of a Chinese crude drug, qiang-huo, the underground parts of $N$. incisum and $N$. forbesii, by highperformance liquid chromatography. Chem Pharm Bull (Tokyo) 38, 2498 - 2502 (1990)

25 Baba K, Yoneda Y, Kozawa M, Fujita E, Wang NH and Yuan CQ: Studies on Chinese traditional medicine Fang-Feng II. Comparison of several Fang-Feng by coumarins chromones and polyacetylenes. Shoyakugaku Zasshi 43, 216-221 (1989) (text in Japanese with English abstract)

26 Kozawa M, Fukumoto M, Matsuyama Y and Baba K: Chemical studies on the constituents of the Chinese crude drug "Quiang Huo". Chem Pharm Bull (Tokyo) 31, 2712 - 2717 (1983)

27 Li RZ, He YQ, Chiao M, Xu Y, Zhang QB, Meng JR, Gu Y and Ge LP: Studies of the active constituents of the Chinese drug "duhuo" Angelica pubescents. Acta Pharm Sin (Yao Hsueh Hsueh Pao) 24, 546-551 (1989) (text in Chinese with English abstract)

28 Berhow M, Tisserat B, Kanes K and Vandercook C: Survey of Phenolic Compounds Produced in Citrus. United States Department of Agriculture, Agricultural Research Service, Peoria, Illinois Technical Bulletin 1856 (1998)

29 Satoh Y, Tashiro S, Satoh M, Fujimoto Y, Xu J-Y and Ikekawa T: Studies on the bioactive constituents of Aurantii Fructus Immaturus. Yakugaku Zasshi 116, $244-250$ (1996) (text in Japanese with English abstract)

30 Tassaneeyakul W, Guo LQ, Fukuda K, Ohta T and Yamazoe Y: Inhibition selectivity of grapefruit juice components on human cytochromes P450. Arch Biochem Biophys 378, 356-363 (2000) 